\title{
Antropologia e práticas artísticas em Portugal
}

Sónia Vespeira de Almeida

CRIA-FCSH ${ }^{1}$

Actualmente os artistas visuais têm vindo a reforçar o diálogo com a antropologia e com os seus métodos. Este artigo procura identificar e examinar as configurações desta relação no quadro de uma investigação sobre a construção da nação nas práticas artísticas em Portugal.

Palavras-chave: discursos e práticas artísticas, antropologia, Portugal

\section{INTRODUÇÃO}

"Uma imagem nunca é uma realidade simples", diz-nos Jacques Rancière (2011 [2003]: 13). Referindo-se às imagens de cinema, sublinha que estas "são antes de mais operações" (2011 [2003]: 13), enfatizando o carácter processual do trabalho artístico e o jogo de relações e de opções que envolve. De facto, as práticas artísticas devem ser perspectivadas como processos que envolvem olhares e interlocuções produtoras de sentidos.

Actualmente, os artistas visuais têm vindo a reforçar o diálogo com a antropologia e com os seus métodos procurando este artigo identificar e discutir as configurações desta relação no contexto de uma investigação sobre a construção da nação nos discursos e práticas artísticas em Portugal (v. Almeida 2012a).

A importância dos nomadismos entre disciplinas tem vindo a ser sublinhada, entre outros, por Garcia Canclini (1997 [1989]) e Mieke Bal (2009 [2002]), que nos têm alertado para a superação das visões e práticas sedentárias. O que aqui proponho é justamente uma análise da expressão destes nomadismos nas artes visuais, tomando como foco de análise a relação entre

1 Faculdade de Ciências Sociais e Humanas, Universidade Nova de Lisboa. O presente artigo insere-se na minha investigação de pós-doutoramento em antropologia intitulada: "Diálogos com a nação nas práticas e discursos da arte contemporânea portuguesa” (Ref. a SFRH/BPD/37456/2007) financiado por Fundos Nacionais através da FCT - Fundação para a Ciência e a Tecnologia. Contato: sonia.almeida@fcsh.unl.pt 
arte e antropologia nas práticas de três artistas portugueses que ilustram de forma enfática e reflexiva o "tráfego" metodológico entre estas duas áreas ${ }^{2}$, orientação que caracteriza actualmente a visualidade em Portugal.

Perceber como pensam os artistas (Garcia Canclini 2005 [1998]) e evidenciar as apropriações da antropologia nas suas práticas e discursos são alguns dos pontos da trajectória que este artigo pretende trilhar.

\section{Cumplicidades}

A identificação e a análise das interlocuções entre arte e antropologia não são recentes. Como mapearam Fernandes Dias (2001), Arnd Schneider e Cristopher Wrigth (2006), é sobretudo a partir da segunda metade da década de 90 do século XX que se assiste à intensificação da problematização das cumplicidades entre estas duas áreas (Marcus e Myers 1995; Foster, 1996; Schneider 1996), numa continuidade com as reflexões lançadas pela designada "antropologia pós-moderna” (Clifford e Marcus 1986, Marcus \& Fischer 1986, Clifford 1988).

Apesar de partilharem terrenos comuns, arte e antropologia têm vindo a desenvolver relações difíceis (Schneider 1996) e vacilantes. Após os debates em torno da arte primitiva e do primitivismo modernista ${ }^{3}$, o foco de discussão orientou-se, também, para a "viragem etnográfica" da arte contemporânea, expressão cunhada pelo crítico de arte Hall Foster no seu célebre texto "The Artist as an Ethnographer" (1996). Segundo Fernandes Dias:

[...] a crítica interna da antropologia acompanha a crítica artística dos primitivismos. Mais do que isso, as questões de identidade e identificação que se tornaram dominantes no trabalho antropológico ocupam também o trabalho artístico. [...] À relação de colaboração e de reciprocidade entre arte e antropologia sucede-se uma situação muito mais complexa, em que ambas tratam dos mesmos assuntos e em que o trabalho artístico adopta frequentemente a atitude do etnógrafo, e às vezes os seus métodos (Dias 2001:116).

Mas as reflexões em torno das interlocuções entre a prática artística e o trabalho antropológico viriam a conhecer contributos posteriores (Schneider e Wrigth 2006, 2010) que procuraram estimular novos diálogos entre a antropologia e arte, sublinhando precisamente as suas afinidades. Estas propostas procuram explorar algumas áreas de intersecção, posicionamentos "in between" (Schneider e Wrigth 2010:2) e encorajar a colaboração e partilha de práticas alternativas nos dois lados da fronteira. Os antropólogos são assim desafiados a estabelecerem relações com o trabalho dos artistas. Aos artistas lança-se o apelo em sentido contrário (Schneider e Wrigth 2006, 2010).

2 Para complemento da informação sobre os artistas analisados, ao longo do artigo são indicadas, sempre que existam, as suas páginas web.

3 Neste quadro, importa sublinhar que a figura do "primitivo" assumiu um papel axial no Modernismo (ver Gill 1993); para análise deste tópico na obra de Lévi-Strauss cf. Passeti, 2008). E será justamente em torno da reflexão sobre esta relação que se assistirá ao reforço do diálogo entre arte e antropologia na década de oitenta. A reintegração deste tópico na agenda antropológica é motivada pela realização no Inverno de 1984 de uma exposição intitulada Primitivism in 20th Century Art: Affinity of the Tribal and the Modern realizada no MOMA em Nova Iorque. "Histories of the Tribal and the Modern", da autoria de James Clifford (1988), é um dos textos paradigmáticos sobre este assunto. Para uma reflexão mais aprofundada sobre a relação entre a arte primitiva e primitivismo ver também Hiller (1991); Price (2001 [1989]). 


\section{Nomadismos}

Escutemos os artistas:

"Eu tinha que ir a África. Há um momento em que digo: - para fazer este projecto sem que eu seja uma mera voyeuse de um processo que eu estou a analisar, a comentar um evento na Europa e na África, conhecendo o lado europeu e não conhecendo o lado africano concreto, ir ao sítio, sentir a temperatura, ir ao sítio falar com as pessoas $[\ldots]^{\prime \prime 4}$ (Ângela Ferreira)

"Eu faço trabalho de campo. Eu, no caso de Nisa, fui tentar perceber quem eram aquelas pessoas." ${ }^{5}$ (Joana Vasconcelos)

"Existe um trabalho de pesquisa que se pode parecer com um trabalho etnográfico de campo, uma vez que para cada projecto existe uma preocupação em confrontar a visão que eu tenho de determinada realidade com essa própria realidade [...]”. (João Pedro Vale in Lapa 2008:24)

$\mathrm{Na}$ minha pesquisa ${ }^{6}$ sobre a ideia de nação nas práticas artísticas contemporâneas em Portugal, o diálogo dos artistas com as ferramentas teórico-metodológicas da antropologia assumiu-se como um tópico incontornável, como evidenciam os discursos dos artistas acima transcritos. Fernandes Dias (2002), ao debruçar-se precisamente sobre estes trânsitos, identifica o seu desenvolvimento em duas direcções:

First, there is the questioning of the traditional ways, anthropological but not exclusively, of dealing with the construction of the cultural other - the "primitive", the collecting of artefacts, its treatment in museums, the representation of others in exhibitions, travelling, etc. [...]. We can find a second trend in the work of artists who use, in their idiosyncratic ways, the ethnographic method of participant observation to engage in localised politics of representation and difference. This is evident in the recurrent ethnographic understanding of site, widening the aesthetic and topographical preoccupations of site-specific art, in adding a social dimension that refers to a human history and memory and using political agendas relevant to a specific place (2002: 206-207).

De facto, na actualidade é possível identificar nas práticas artísticas em Portugal uma matriz antropológica que expressa, sobretudo, a segunda tendência identificada por Fernandes Dias (2002), nomeadamente o desenvolvimento de uma atitude de conhecimento aprofundado sobre os contextos culturais mobilizados, a aproximação à perspectiva emic e o recurso ao método etnográfico. Mas estas sobreposições surgem, também, associadas a uma reflexão sobre os tópicos que constituem a agenda da antropologia contemporânea - globalização, emigração, identidades nacionais, cultura popular, pós-colonialismo, género e poder - o que parece aproximar os artistas do antropólogo at home (Dias 2001), tomando a sua própria cultura como objecto de interpelação e crítica. Este posicionamento remete para o argumento expresso por Marcus e Myers (1995) em The Traffic in Culture. Refiguring Art and Anthropology de que ambos - arte e antropologia - convocam a "cultura" como objecto, num movimento de "regresso do real" como lhe chamou Hal Foster (1996).

\footnotetext{
$4 \quad$ Entrevista A.F., 2008.

5 Entrevista J. V., 2009.

6 Iniciada em 2008, a pesquisa mobilizou a uma estratégia múltipla relacionando vários métodos de pesquisa: a entrevista em profundidade junto de artistas, observação directa e pesquisa documental.
} 


\section{Ângela FerReira: "Só a Forma não chega para mim"}

A prática artística de Ângela Ferreira (Moçambique, 1958) é reveladora deste posicionamento. Segundo Gertrud Sandqvist "Ângela Ferreira uses her art as a way of asking questions about her own reality, about its history, its politics, its memories. Starting from her own portion of the world, she treats her art like a wide-angle lens" (Sandqvist 2007: 21). Para a escultora, a proposta de Hall Foster em "The Artist as an Ethnographer" - foi "um texto vital" para trabalhar a temática central da sua obra que identifica como sendo "as relações culturais, as relações de identidade entre África e a Europa"'. Neste sentido, Ângela Ferreira trabalha, também, com os conceitos de cultura, contexto cultural, alteridade e identidade, pedindo de empréstimo à antropologia as suas ferramentas conceptuais.

O início do percurso artístico de Ângela Ferreira passou pelo diálogo com a cultura popular urbana no contexto da sua dissertação de mestrado intitulada An investigation into a relationship between personal sculptural statement and popular material culture (1984). Num documento que integra o seu processo de candidatura a uma bolsa de estudo do Serviço de Belas Artes da Fundação Calouste Gulbenkian (Portugal), a artista afirma:

\footnotetext{
O meu trabalho tem estado intimamente ligado à cultura popular. Sempre trabalhei baseada na observação das imagens do mundo que me rodeia. [...] Após ter terminado a tese em 1984, o meu trabalho tomou um rumo mais específico no que diz respeito à escolha de referência artística. Como é óbvio, passei a observar com mais curiosidade a cultura popular sul-africana e africana. Foi também por esta altura, numa das visitas a Portugal, que descobri pela primeira vez elementos da cultura portuguesa que me interessaram bastante e comecei a desenhá-los. ${ }^{8}$
}

Em 1989, recebe a bolsa de estudos à qual se candidatara e inicia a sua indagação em torno do "sentido da identidade portuguesa". O ponto de partida de Ângela Ferreira são os objectos de cultura material de matriz rural, em particular os arados, que integravam o acervo do Museu Nacional de Etnologia em Lisboa ${ }^{10}$.

O contexto cultural a partir do qual resgata as suas referências é fundamental no processo de criação artística da escultura, afirmando que "só a forma não chega para mim”. Ângela Ferreira procura, assim, que o seu trabalho tenha uma "empatia com todo o mundo".

A artista revela igualmente um fascínio pela arquitectura, especialmente na sua expressão modernista como ilustra Maison Tropical (2007) ${ }^{11}$. Esta instalação decorre da sua discussão, iniciada em 1997, sobre a política cultural em Africa, a história e memória colonial e as suas

$7 \quad$ Entrevista a A.F., 2008.

8 Anexo I ao Boletim de Inscrição para Bolsa de Estudo de Especialização, 1989, Serviço de Belas Artes, Arquivo SBA, Proc. N. 4894.

9 Relatório intitulado Fase Final - projecto baseado nos painéis "Emigração", de Almada Negreiros situados na Gare Marítima Rocha de Conde de Óbidos, que inclui também a produção da exposição "Portugal dos Pequenitos", 1996, Arquivo SBA, Proc. N. 7168.

10 Este projecto deu origem à exposição $A$ Propósito de..., realizado no Centro de Arte Moderna José Azeredo Perdigão, em Lisboa, no ano de 1990.

11 Esta instalação foi a sua proposta para representar oficialmente Portugal na 52a Exposição Internacional de Arte da Bienal de Veneza (2007). 
ramificações contemporâneas. Ângela Ferreira sublinha que é a dimensão antropológica - que reconhece no seu trabalho - que a conduz ao diálogo com a arquitectura:

[...] porque a arquitectura é um objecto de referência ideal no sentido em que é popular, é usado, é social, é antropológico, é tudo. E depois também é formal, é tridimensional e também é escultórico. Não está no âmbito do arado como objecto etnográfico, mas podemos olhá-lo como tal. E é por aí que eu pego na arquitectura, mais uma vez incorri em muitos desgostos porque as pessoas pensavam que eu recorria à arquitectura só em termos formais, mas não. ${ }^{12}$

Quer nos projectos iniciais em torno da cultura popular de origem rural, quer em Maison Tropicale, Ângela Ferreira convoca o método etnográfico. Relativamente aos seus primeiros trabalhos coloca a ênfase no estudo aprofundado da colecção do Museu de Etnologia:

Tal como havia planeado, comecei por investigar e documentar imagens e textos do assunto que me compete. Para esse fim tinha decidido viajar pelo país para documentar elementos e objectos de arte popular. No entanto, durante os primeiros passos que dei nesse sentido em Lisboa, encontrei nas bibliotecas e nos museus da cidade e muito especialmente no Museu de Etnologia, uma colecção riquíssima de objectos de arte popular. Decidi então começar o projecto sem optar imediatamente pelas viagens e com a simpática ajuda e valiosos conhecimentos do $\mathrm{Sr}$. Benjamim Pereira. [...] A minha proposta de trabalho esboçada é portanto de detectar algumas áreas de arte popular escolhidas tanto pela importância dos objectos referenciados e pela sua profusão no país, como pela minha reacção à sua qualidade estética e possibilidade de potencial escultórico (formal e de conteúdo). As áreas escolhidas foram: os jugos, o carro de bois, as alfaias agrícolas, os gatos de chaminé, os transfogueiros e os objectos de cortiça do Alentejo (pela originalidade de material). ${ }^{13}$

O pendor antropológico percorre de forma continuada a sua obra e manifesta-se quer ao nível das temáticas, quer dos recursos metodológicos convocados. Esta última dimensão foi captada por Manthia Diawara em Maison Tropicale (2008). O realizador descreve, deste modo, o trabalho de Ângela Ferreira em Niamey:

Ângela began interacting with the Tuareg women right way. She walked on the terrace with them and asked questions through body and hand gestures. Suddenly I realised that she had brought the house back to the present, back to life, through the memories of one woman named Artannor Ibriahine. As she asked Artannor Ibriahine where the kitchen, the living room, and the other parts of the house had been, Ângela was at the same time entering with conversation with Prouvé. (Diawara 2007: 49-52)

Neste documentário é possível observar a artista em trabalho de campo em Niamey e Brazzaville. Ângela Ferreira entrevista os diferentes agentes locais procurando perceber como as casas foram vividas por quem as habitou, perscrutando as memórias. Mas a escultora procura, também, traçar a viagem das Casas Tropicais desenhadas na Europa pelo arquitecto modernista Jean Prouvé, transportadas para África (Níger e a República do Congo) e, novamente, transferidas para a Europa onde circulam no mercado de arte internacional. Referindo-se ao processo de criação associado a este projecto, Ângela Ferreira sublinha que envolveu muita investigação em torno do percurso biográfico destas construções, destacando que fez "muito trabalho de campo": "Eu queria entender onde as casas estavam e como as casas estavam. Eu queria ver o local. Eu queria documentar os despojos, essa era a minha intenção." ${ }^{14}$

12 Entrevista A.F., 2008.

13 Relatório $10^{\circ}$ Trimestre Projecto de Escultura, 1989 SBA, Proc. N. 3249.

14 Entrevista A.F., 2008. 
Também a prática artística de Joana Vasconcelos ${ }^{15}$ (Paris, 1971) manifesta uma matriz antropológica, partilhando a sedução pelo "outro" que procura dotar de sentido. A artista tem vindo a mobilizar um repertório "etno-cultural" (Leoussi 2004), discutindo e reflectindo sobre ideia de nação, cimentando o seu trabalho nos estereótipos e símbolos nacionais e regionais.

Aquando da realização da entrevista, a artista preparava-se para inaugurar a peça Valquíria Enxoval (2009) instalada no Complexo Termal da Fadagosa em Nisa. Este trabalho foi o resultado de uma proposta do município desta vila que lhe lançou o repto de trabalhar a partir do artesanato desta região alentejana, em particular a partir dos têxteis e bordados que constituíam os enxovais das noivas ${ }^{16}$. Valquíria Enxoval - que integra a série Valquírias (2004-2009) - é uma estrutura flexível pendurada no tecto cujas componentes são "alinhavados e outros bordados, aplicações em feltro, rendas de bilros, frioleiras, olaria pedrada, tricô e croché em lã feitos à mão, tecidos adereços, poliéster e cabos de aço" (Amado 2010: 31).

Visitou Nisa várias vezes. Referindo-se à relação com os agentes locais, Joana Vasconcelos refere de forma detalhada a importância da aproximação, da negociação exigente e do conhecimento aprofundado dos contextos culturais: "Muito depressa tive que entender quem era quem. Qual era o bom atelier, qual era a especialista dos bordados, qual era a especialista do ponto grilhão, qual era a especialista dos alinhavados, qual a especialista dos feltros, porque os grupos são variados."17

Referindo-se ao processo de criação de Valquíria Enxoval, sublinhou a importância da sua presença nos ateliers, da observação do gesto técnico, das conversas com as artesãs, revelando um fascínio pelas suas histórias de vida e pelas identidades de género. Joana Vasconcelos explica, do seguinte modo, como a "investigação no terreno" integra a sua prática: "Quando entras nas coisas não há nada a fazer. Começas a aprender, a distinguir a olhar, a perceber como são as pessoas, o que as pessoas têm na cabeça, quais são as influências e, depois, é um gosto"18.

Para Amado (2010), Joana Vasconcelos revela uma sintonia com a "viragem etnográfica” na arte proposta por Foster (1996), sugerindo que as suas propostas não divergem muito das dos artistas da sua geração ao fomentar um debate em torno das questões de identidade inseridos numa análise crítica da contemporaneidade.

\section{João Pedro Vale: "Interessa-me perceber como as pessoas sentem"}

Are you still awake? é o nome de uma exposição inaugurada no final de 2012 no Museu Nacional de Arte Contemporânea, Museu do Chiado em Lisboa que pretende assinalar a relação entre arte e política na visualidade portuguesa. Esta interrogação foi retirada de uma obra de

15 Ver http://www.joanavasconcelos.com. Joana Vasconcelos é artista que representará Portugal na 55a Exposição Internacional de Arte da Bienal de Veneza em 2013.

16 No âmbito deste projecto foi realizado um documentário intitulado Enxoval (2010), que acompanha o processo de criação desta peça.

17 Entrevista a J. V., 2009.

18 Entrevista a J.V., 2009. 
João Pedro Vale ${ }^{19}$ (Lisboa, 1976) com o mesmo título e revela, justamente, a importância do comentário crítico relativamente à contemporaneidade que o artista tem vindo a desenvolver, recorrendo a técnicas e meios diversificados.

O artista opera recortes na realidade, trabalha a partir do micro, afirmando "isto é mais próximo do que vocês [antropólogos] fazem. [...] Para mim é mais fácil de analisar"20. João Pedro Vale circunscreve as realidades que lhe estão mais próximas culturalmente para trabalhar os temas centrais do seu trabalho - a identidade sexual e nacional (Lapa 2008) - combinando "as qualidades formais de um dado material e contexto (conforme o projecto) para criar trabalhos que funcionam quase como locais discursivos" (Sharp 2008:101).

A emigração açoriana para os Estados Unidos da América tem-se constituído o foco da sua interpelação nos últimos anos, como demonstram os projectos Moby Dick (2009), English As She Is Spoke (2010) e, mais recentemente, a instalação P-Town (2012) realizada em parceria com Nuno Alexandre Ferreira.

O projecto Moby Dick ${ }^{21}$, estruturado a partir da obra homónima de Herman Melville (1851), desdobra-se numa série de trabalhos que mobilizam técnicas diferenciadas (filme, instalação, pintura, desenhos, construção de objectos). $\mathrm{O}$ artista investigou o movimento migratório dos portugueses oriundos do arquipélago dos Açores que residiram na costa leste dos Estados Unidos da América, em particular os pescadores baleeiros. $\mathrm{O}$ artista explica, deste modo, o processo de criação associado a Moby Dick:

Eu quando estive em Nova Iorque estive a trabalhar sobre Moby Dick. Fui trabalhar sobre Moby Dick para poder falar sobre os emigrantes porque havia uma presença tão forte dos Açores nos Estados Unidos, é como se o Moby Dick fosse o elo de ligação entre a cultura americana e os Açores. [... $]^{22}$

Posteriormente João Pedro Vale visitou este arquipélago após ter terminado o projecto English As She Is Spoke ${ }^{23}$, trabalho subsequente que mantém como horizonte de interlocução os Açores, que viria a ser retomado em $P$-Town:

Tenho necessidade de ir aos Açores porque de repente estou a falar de uma realidade que existe e que nós temos acesso. Sinto que tenho que ir lá, não tanto para perceber se retratei de forma exacta, porque o meu objectivo não era retratar aquilo, mas utilizar. [...] Interessa-me ir lá para perceber como aquelas pessoas sentem. ${ }^{24}$

João Pedro Vale reconhece o diálogo com a antropologia na sua prática, em particular ao nível do método que utiliza, marcado pelo recurso ao trabalho de campo e à forte interlocução com os agentes locais ${ }^{25}$. Contudo, evidencia as diferenças e desencontros entre a prática artística e a prática antropológica, afirmando: "Eu faço investigação, mas não tenho uma preocupação

19 Ver www.joaopedrovale.com

20 Entrevista a J.P.V., 2010.

21 Este projecto foi o resultado de uma residência artística de sete meses em Nova Iorque (2008-2009) no âmbito do International Studio and Curatorial Program.

22 Entrevista a J.P.V., 2010.

23 Este projecto integra um filme, um conjunto de esculturas e desenhos.

24 Entrevista a J.P.V., 2010.

25 A relação com os agentes locais é particularmente evidente em Ala Arriba! (2006) no âmbito do qual trabalha directamente com um grupo de folclore na Nazaré. 
científica. Não é científica no sentido de tentar ver todas as dimensões do problema”, sublinhando, ainda, que o seu trabalho:

[...] Em todo caso não é etnográfico no sentido em que não inicio essa pesquisa com a pretensão da página em branco ou de me despir das minhas próprias concepções para averiguar uma suposta verdade. Inclusivamente interessam-se os meus preconceitos como ponto de partida para a investigação. $\mathrm{O}$ que eu procuro no campo não é desfazê-los, mas tentar perceber a forma como esses agentes locais contribuem para a sua formulação. Tal como no trabalho etnográfico existe uma recolha de dados em relação aos quais eu não tenho qualquer prurido, mas posso atribuir-lhes diferentes graus de importância consoante a orientação que pretendo dar ao meu projecto [...]. (in Lapa 2008: 24)

As palavras de João Pedro Vale são particularmente reveladoras por um lado, dos usos idiossincráticos da antropologia (Schneider, 1996) pelos artistas e, por outro, das representações que possuem da disciplina. Como sublinhou Fernandes Dias (2001), a partir das reflexões de Foster e Schneider, no trabalho artístico "quase-antropológico" (Foster 1996), os princípios da observação participante não são muitas vezes respeitados, bem como a duração da estadia, aludindo, ainda, aos níveis de profundidade e de sistematização dos usos da antropologia pela arte.

\section{RefleXões finais}

O diálogo entre arte e antropologia tem assumido denominações várias - "Etnografia espontânea" (Leal 2000), "sensibilidade antropológica" (Almeida 2009, 2012b) ou "antropologia paralela” (Tentori cit. in Feixa 2008) - expressões que procuram justamente expressar a sedução de um conjunto de agentes - oriundos de áreas e contextos diferenciados - pela antropologia.

Ao longo deste texto procurei precisamente evidenciar e problematizar a interlocução da visualidade portuguesa contemporânea com as ferramentas e a agenda da antropologia contemporânea, identificando a sobreposição de temáticas e uma predisposição para a utilização do método etnográfico nas suas diferentes dimensões.

No caso da presente investigação, os recursos da antropologia são mobilizados nas práticas artísticas no contexto da construção de um discurso crítico sobre o mundo contemporâneo. Como procurei demonstrar através dos exemplos convocados, a "nação" surge como uma plataforma que permite revelar posicionamentos vários face ao colonialismo e pós-colonialismo, à globalização, às migrações e identidades. Aqui encontramos as zonas de sobreposição. Os artistas operam recortes na realidade - experimentando o método etnográfico - e expressam análises críticas através dos seus projectos, posteriormente difundidos pelos circuitos do campo artístico.

Em 2010, Néstor Garcia Canclini proferiu uma lição em Lisboa no âmbito do projecto "Próximo Futuro" da Fundação Calouste Gulbenkian. O antropólogo afirmou que a antropologia, disciplina que partilha saberes de outras disciplinas, tem que estar "atenta ao que chega". Respondendo ao repto do antropólogo, importa continuar a interrogar quais as contribuições mútuas da arte e da antropologia contemporâneas ${ }^{26}$.

26 Recentemente, Alban Bensa e François Pouillon organizaram a obra Terrains d'Écrivains. Littérature et 
Há mais de duas décadas que a antropologia deu início à problematização deste movimento nas artes visuais, análise não isenta de alguma suspeição (Schneider 1996), na medida em que estas apropriações ameaçavam a sua "pureza" e integridade disciplinar. Acompanhando Fernandes Dias (2001) e Schneider e Wrigth $(2006,2010)$, considero que o trabalho antropológico e o trabalho artístico podem iluminar-se mutuamente se se orientarem na superação de alguma tensão e ansiedade disciplinar.

Para finalizar, e numa inversão da perspectiva argumentativa do presente artigo, assumese como pertinente estender a problematização aos usos da arte pela antropologia e perceber as potencialidades do diálogo nesta direcção. Se a antropologia visual ajudou a contrariar alguma tendência iconofóbica da antropologia, pouca atenção tem sido dirigida à possibilidade de mobilização de outras formas de expressão nos processos de produção do conhecimento antropológico, nomeadamente ao papel da pintura, como sublinhou Ossman (2010):

Much less attention has been paid to the ways that the practice of other kinds of aesthetic practice can play in the development of anthropological knowledge. While at least some attention has been given to musical performance, to theatre or to the poems of sketches of anthropologists, the possibility of painting in the field has been pointedly ignored. (2010: 127)

Acredito que o caminho nesta direcção trará contributos sólidos à antropologia ao sugerir, por exemplo, novas formas de registo dos dados etnográficos - ou à recuperação de outras como o desenho etnográfico (v. Afonso 2004) - e, ainda, de transmissão e difusão do conhecimento antropológico.

\section{Biblografia}

Afonso, Ana I.. 2004. "New graphics for old stories. Representation of local memories through drawings" (com desenhos de Manuel João Ramos). Pp. 72-89 in Working Images, Visual Research and Representation in Ethnography edited by S. Pink, A.I Afonso, K László, London, New York: Routledge.

Almeida, Sónia V., 2009, Camponeses, Cultura e Revolução. Campanhas de Dinamização Cultural e Aç̧ão Cívica do MFA (1974-1975), Lisboa: IELT -Colibri.

_ 2012a. "Que nação é esta? Tramas identitárias nas artes visuais portuguesas». Visualidades. Vol 10, N. o 1, Programa de Pós-graduação em Cultura Visual - Mestrado da Faculdade de Artes Visuais da Universidade Federal de Goiás - Brasil: 39- 61

2012b. «Gaibéus: "antes de tudo um documentário humano". Povo, arte e etnografia». Comunicação apresentada no colóquio Alves Redol e as Ciências Sociais - a literatura e o real, os processos e os agentes, Lisboa, 7-10 de Novembro 2012.

Amado, Miguel. 2010. "Ponto de Encontro ou o Regresso da Arte do Real”. Pp. 15-51 in Joana Vasconcelos. Sem Rede, Lisboa: Museu Colecção Berardo.

“Ângela Ferreira, A Propósito de” [catálogo de exposição]. 1990. Lisboa: Fundação Calouste Gulbenkian/Centro de Arte Moderna.

Bal, Mieke. 2009 [2002]. Conceptos Viajeros en las Humanidades. Una guia de Viaje. Múrcia: CENDEAC.

Ethnographie (2012) que sinaliza precisamente a fecundidade e a necessidade da reflexão sobre as afinidades entre os processos de criação artística e a antropologia. 
Bensa, Alban, Pouillon, François (eds.). 2012. Terrains d'Écrivains. Littérature et Ethnographie. Toulouse: Anacharsis.

Clifford, James; Marcus, George (ed.). 1986. Writing Culture: The Poetics and Politics of Ethnography. Berkeley: University of California Press.

Clifford, James. 1988. The predicament of culture: twentieth-century ethnography, literature and art. Cambridge: Harvard University Press.

Dias, José A. F. 2001. "Arte e Antropologia no século XX: Modos de relação", Etnográfica, Vol. V (1), CEAS/Celta: 103-129

- 2002. "The Ethnographic Turn as a Method of Analysis in Contemporary Art Practice". Pp 203-333 in From work to Text. Dialogues on Practise and Criticism in Contemporary Art, edited J. Bock, Lisboa: Fundação Centro Cultural de Belém.

Diawara, Manthia. 2007. "Architecture as Colonial Discourse Ângela Ferreira: On Jean Prouvé's Maisons Tropicales". Pp. 38-53" in Maison Tropicale, Angela Ferreira. edited J. Bock , Lisboa: Instituto das Artes/Ministério da Cultura.

Feixa, Carles. 2008. "Más allá de Éboli: Gramsci, De Martino y el debate sobre la cultura subalterna en Itália”. Pp. 13-66. in El folclore progresivo y otros ensayos, Barcelona: Editorial MACBA / UAB-Universitat Autònoma de Barcelona.

Foster, Hall. 1996. The Return of the real, The Avant-Garde at the End of the Century, Cambridge, Massachusetts, London: The MIT Press.

Garcia Canclini, N. 1997 [1989]. Culturas Hibridas, Estratégias para entrar e sair da Modernidade. São Paulo: Editora da Universidade de São Paulo.

2005 [1998]. "Remaking Passports. Visual Thought in the debate of multiculturalism", Pp. 180-189 in The Visual Culture reader edited by N. Mirzoeff. London: Routledge.

Gill, Perry. 1993."Primitivism and the 'modern".Pp.3-86 in Primitivism, Cubism and Abstraction, The Early Twentieth Century. C Harrison, F. Frascina, P. Gill. New Haven, London: Yale University Press.

Hiller, Susan. (eds.). 1991. The Myth of Primitivism. Perspectives on Art. London, New York: Routledge.

Lapa, Pedro. 2008. "O Vício da História. Pedro Lapa em conversa com João Pedro Vale”. Pp. 10-99 in Nascido a 5 de Outubro, Lisboa: ADIAC Portugal, Tugalandia.

Leal, João. 2000. Etnografias Portuguesas (1870-1970), Cultura Popular e Identidade Nacional. Lisboa: Publicações D. Quixote.

Leoussi, Athena. S. 2004. "The ethno-cultural roots of national art". Nations and Nationalism, $10(1 / 2): 143-159$.

Marcus, George, Fischer, M. 1986. Anthropology as Cultural Critique: An Experimental Moment in the Human Sciences. Chicago: The University of Chicago Press.

Marcus, George, Meyers, Fred R., (eds.). 1995. The Traffic in Culture. Refiguring Art and Anthropology. Berkeley, Los Angels, London: University of California Press.

Ossman, Susana. 2010. "Making Art Ethnography: Painting, War and Ethnographic Practice". Pp. 127-134 in Between Art and Anthropology. Contemporary Ethnographic Practice edited by A. Schneider, C. Wright, Oxford, New York: Berg.

Passetti, Dorothea V.. 2008. Lévi-Strauss, antropologia e arte: minúsculo - incomensurável, São Paulo: EDUSP, EDUC.

Price, Sally. 2001 [1989]. Primitive Art in Civilized places. Chicago: University of Chicago Press. Rancière, Jacques, 2011 [2003]. O destino das imagens. Lisboa: Orfeu Negro.

Sandqvist, Gertrud. 2007. "Sculpture Revisited”, Pp. 20-34 in Maison Tropicale, Angela Ferreira. Edited by J. Bock, Lisboa: Instituto das Artes/Ministério da Cultura. 
Schneider, Arnd. 1996. “Uneasy relationship: Contemporary artists and Anthropology”. Journal of Material Culture, 1 (2): 183-210.

2006. “Appropriations". Pp. 29-51 in Contemporary Art and Anthropology edited by edited by A. Schneider, C. Wright. Great Britain: Berg.

Schneider, Arnd; Wright, Christopher (eds.). 2006. Contemporary Art and Anthropology. Oxford, New York: Berg.

2010. Between Art and Anthropology. Contemporary Ethnographic Practice. Oxford, New York: Berg.

Sharp, Chris. 2008. "Arte e Mentiras”. Pp. 99-126 in Nascido a 5 de Outubro. Lisboa: ADIAC Portugal, Tugalandia.

\section{FILMOGRAFIA}

Diawara, Manthia. 2008. Maison Tropicale, Portugal, 58'. Produção: Maumaus-Escola de Artes Visuais

\section{FONTES ARQUIVÍSTICAS}

Fundação Calouste Gulbenkian - Serviço de Belas Artes

Processos: N. 4894 ; N.o 7168 , N.o 3249.

\section{Anthropology and artistic practices in Portugal}

In Portuguese visual arts one can observe a reinforcement of the dialogue with anthropology and its methods. This article aims to examine this dialogue, based on research about the idea of nation present in contemporary Portuguese art practices.

Keywords: artistic practices and discourses, anthropology, Portugal 